\title{
POSSIBLE COMETARY ORGANIC COMPOUNDS AS SOURCES OF PLANETARY BIOSPHERES
}

\author{
Kensei Kobayashi ${ }^{1,2}$, Yoshinori Takano ${ }^{1}$, Hitomi Masuda ${ }^{1}$, Hisako Tonishi ${ }^{1}$, Takeo Kaneko ${ }^{1}$, \\ Hirofumi Hashimoto ${ }^{3}$, Takeshi Saito ${ }^{4}$ \\ ${ }^{1}$ Department of Chemistry and Biotechnology, Yokohama National University, Yokohama 240-8501, Japan \\ ${ }^{2}$ Institute of Space and Astronautical Science, Sagamihara, 229-8510, Japan \\ ${ }^{3}$ Institute of Engineering Mechanics, University of Tsukuba, Tsukuba 305-8573, Japan \\ ${ }^{4}$ Institute for Advanced Studies, 1-29-6 Shinjuku, Shinjuku-ku, Tokyo 160-0022, Japan
}

\begin{abstract}
Various organic compounds including complex organic compounds have been detected in comets. Cometary organics are supposed to be formed in interstellar dust (ISD) environments. We examined possible formation of bioorganic compounds in ISDs through simulation experiments, and compared with that in primitive Earth atmosphere. It was shown that a wide variety of amino acids were formed in simulated ISD environments in the form of precursors. Pyrimidine bases (uracil, cytosine and thymine) were also formed from the possible interstellar media. These results suggest that comets can bring no less types of bioorganics to planets than those formed in primitive Earth atmosphere. Not only Earth, but also other planetary bodies like Mars and Europa received cometary organics during the late heavy bombardment era. They can be included in possible habitable areas whatever primitive planetary atmosphere they had.
\end{abstract}

\section{INTRODUCTION}

The first requisite for the generation of life on planets is supply of organic compounds as well as the presence of liquid water. Since Miller (1953)'s historical experiment, a number of studies have been done to show that bioorganic compounds including amino acids are easily formed if the planet has a reducing atmosphere containing methane and nitrogen or ammonia. Nowadays we estimate that the primitive earth atmosphere is not strongly reducing, but only slightly reducing: It was composed of carbon dioxide, carbon monoxide, nitrogen and water (Kasting, 1990). It is much difficult to synthesize organic compounds, particularly nitrogen-containing bioorganic compounds from such gas mixtures (Schlesinger and Miller, 1983). Cosmic rays can assist to form bioorganic compounds from that kind of less reducing atmospheres (Kobayashi et al., 1998; Miyakawa et al., 2002): The amount of endogenously-formed bioorganic compounds would be, however, much less than that previously expected from the strongly-reduced atmosphere.

The alternative source for the first planetary biosphere is exogenous delivery. Carbonaceous chondrites, comets and interplanetary dust particles are three of the major candidates, since they contain considerable amount of organic compounds. Chyba and Sagan (1992) estimated the inventory rates of organic (or carbon-containing) compounds by meteorites, comets and IDPs, and concluded that approximately $6 \times 10^{7} \mathrm{~kg}$ of organic carbon was delivered by extraterrestrial bodies (mainly by IDPs). It should be noted that all of the "organic carbons" could be used for the first living organisms. Thus it is of interest to see what types of bioorganic compounds the extraterrestrial bodies carry.

It has been reported extracts of some types of carbonaceous chondrites such as Murchison meteorite contain a wide variety of amino acids (Kvenvolden et al., 1970) and some nucleic acid bases (Stocks and Schwartz, 1981). Mass spectra of Halley's comet suggested that cometary dusts have various types of organic compounds (Kissel and Krueger, 1987). There were, however, no indications for $\alpha$-amino acids in cometary dusts. Then could comets bring bioorganic compounds to the primitive earth? 
It has been hypothesized that cometary organic compounds were formed in interstellar dust particles (ISDs) (Greenberg and $\mathrm{Li}, 1997$ ). A great number of experiments have been done simulating the condition of ISD environments. Amino acids were reported to form in simulated ISD environments by proton irradiation (Kobayashi et al., 1995; Kasamatsu et al., 1997a) and by UV irradiation (Briggs et al., 1992; Kobayashi et al., 1999; Bernstein et al., 2002; Munos Caro et al., 2002) of simulated ISD ice mantles by using cryostats. It is reported that amino acids were detected in the hydrolysates of the products of ISD-simulating experiments. It was quite difficult, however, to tell the formation rates of bioorganic compounds in ISD environments due to the limitation of experiments. Here we discuss nature of bioorganic compounds formed in simulated ISD environments, and compare with those formed in simulated primitive planetary atmospheres. Starting materials selected are methanol, ammonia and water, all of which are molecules detected in both comets and ISDs. Energies applied to the materials are $\gamma$-rays and ultraviolet light: The former can penetrate into the materials deeply, and the latter affects only the surface of the materials. The starting mixture was placed in a liquid nitrogen bath (77K), in a cold water bath (293K), or in a hot water bath (353K), so that the starting material was kept as solid ice, liquid or gas, individually.

\section{EXPERIMENTAL}

\section{Chemicals}

Deionized water was further purified with a Millipore Milli-Q Labo System and with a Millipore Simpli Lab-UV (both Japan Millipore Ltd., Tokyo, Japan), successively in order to eliminate inorganic and organic impurities. Methanol and ammonia used were of ultra pure grade. In typical experiments, a mixture of methanol, ammonia and water was used as simulated interstellar media (SIM), whose molar ratio was 1 (methanol): 1 (ammonia): 2.8 (water). All the glasswares used were heated in a Yamato DR-22 high temperature oven at $773 \mathrm{~K}$ in prior to use for the elimination of organic contaminants.

\section{$\gamma$-Irradiation of SIM}

SIM placed in a Pyrex tube $(50 \mathrm{~mm}$ i.d. x $150 \mathrm{~mm})$ was subjected to irradiation with $\gamma$-rays $(1.2-1.3 \mathrm{MeV})$ from a ${ }^{60} \mathrm{Co}$ source. The three phases of the SIM were achieved as follows: The solid SIM was made in liquid nitrogen bath to keep at $77 \mathrm{~K}$. The liquid SIM was irradiated at ambient temperature. The gaseous SIM was prepared by heating liquid SIM by heating with a heating tape. Three types of SIM were irradiated at the ${ }^{60} \mathrm{Co}$ facility in Research Center for Nuclear Science and Technology, University of Tokyo. Dose rate, which was measured with a Fricke dosimeter (Ikeda and Suzuki, 1998), was in the range of 4-1600 Gy $\mathrm{h}^{-1}$. In typical experiments, the target was placed at $15 \mathrm{~cm}$ from the ${ }^{60} \mathrm{Co}$ source for $2 \mathrm{~h}$, where the dose rate was $215 \mathrm{~Gy} \mathrm{~h}{ }^{-1}$. In this case, the total energy deposit to the target mixture (SIM) of $8.65 \mathrm{~g}$ was $2.3 \times 10^{19} \mathrm{eV}$.

\section{UV Irradiation of SIM}

The same kinds of the starting materials for UV-irradiation in solid and liquid phases were placed in a quartz tube $(40 \mathrm{~mm}$ i.d. x $10 \mathrm{~mm}$ ); A Pyrex tube $(50 \mathrm{~mm}$ i.d. x $200 \mathrm{~mm}$ ) with a synthesized quartz window (30 mm $\phi$ ) was used for UV-irradiation in gas phase. A $150 \mathrm{~W}$ deuterium lamp with a $\mathrm{MgF}_{2}$ window (Hamamatsu Photonics L1835) was used for irradiation. Only UV light whose wavelength was over $160 \mathrm{~nm}$ could pass through the quartz window into SIM. The energy deposit to the SIM in the typical experiments was $3.1 \times 10^{20} \mathrm{eV}$ each.

\section{Determination of Amino Acids}

After irradiation, an aliquot of the irradiation products was hydrolyzed with $6 \mathrm{M} \mathrm{HCl}$ at $110{ }^{\circ} \mathrm{C}$ for 24 hours. Amino acids in the hydrolyzed and unhydrolyzed fraction were analyzed with an ion exchange high performance liquid chromatography (HPLC) system where a post-column derivatization with o-phthalaldehyde and N-acetyl-L-cystein was applied (Kobayashi et al., 1991). The HPLC system used was composed of two liquid chromatograph pumps (Shimadzu LC-6A), a cation exchange column (Shimpak ISC-07/S1504, 4 mm i. d. $\times 150$ $\mathrm{mm}$ ), a post column derivatization system, and a Shimadzu RF-535 fluorometric detector. Identification of amino acids was done by comparing the retention time of the detected peaks with that of authentic standards.

\section{RESULTS AND DISCUSSION}

Formation of Amino Acid Precursors by $\gamma$-Irradiation 


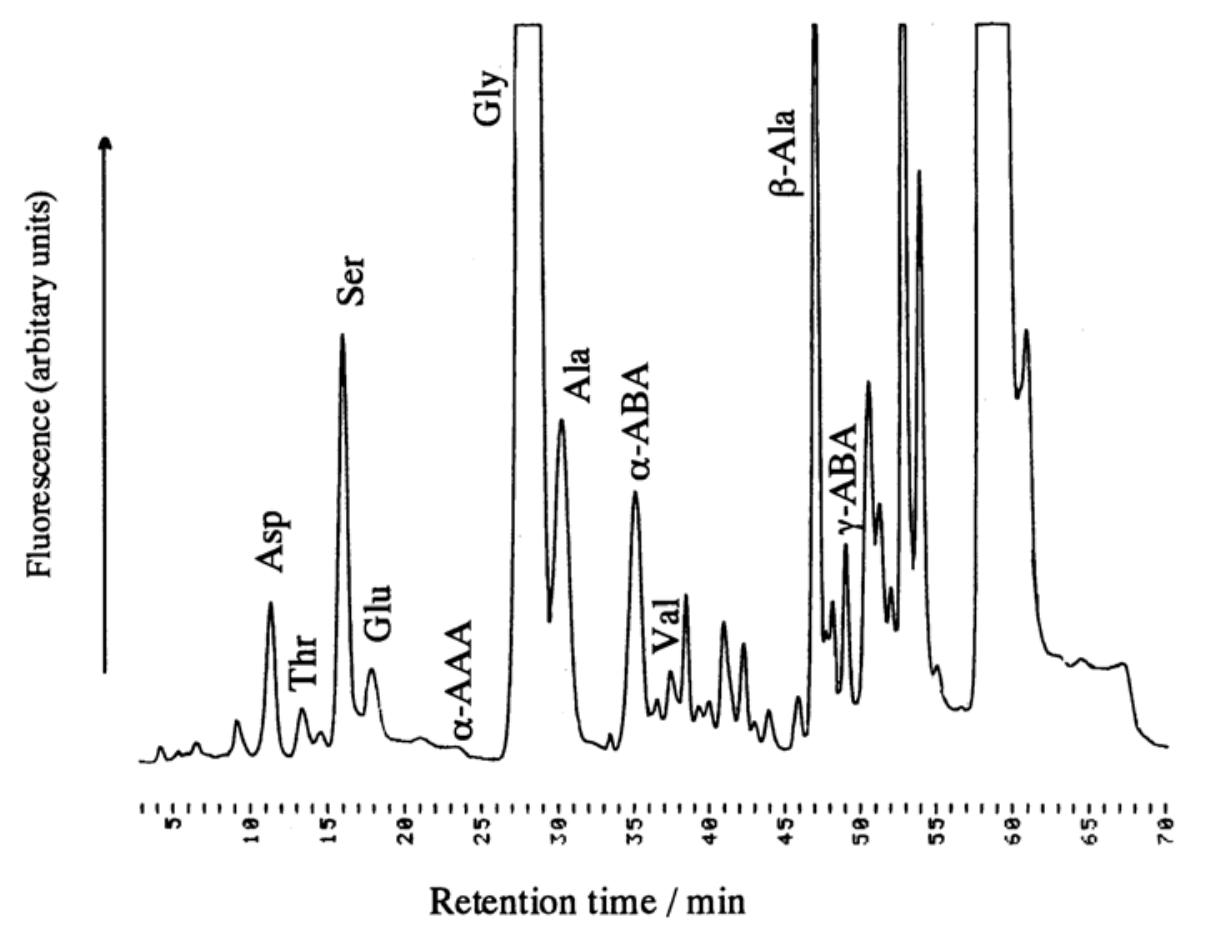

Fig. 1. HPLC chromatogram of amino acids by UV irradiation of icy mixture of methanol, ammonia and water. The irradiation product was hydrolyzed before analysis. Abbreviations: Asp: aspartic acid, Thr: threonine, Ser: serine, Glu: glutamic acid; AAA: aminoadipic acid; Gly: glycine, Ala: alanine, ABA: aminobutyric acid, Val: valine.

Figure 1 shows typical HPLC chromatograms of the $\gamma$-irradiation products. An ion-exchanged chromatogram of the product of UV irradiation of the gas mixture of methanol, ammonia and water was shown in Figure 1. A wide variety of proteinous amino acids such as glycine, alanine, aspartic acid and non-proteinous amino acids such as $\beta$-alanine, $\alpha$ - and $\gamma$-aminobutyric acid were detected. The presence of non-protein amino acids indicated that the amino acids found were not contaminated but indigenous to the product. In the unhydrolyzed fraction, only small amount of glycine was detected. It showed that not free amino acids, but amino acid precursors were formed during irradiation.

\section{Formation of Amino Acid Precursors by UV Irradiation}

UV irradiation of the SIM gave similar results as the $\gamma$-irradiation: Hydrolysates of the products gave a wide variety of amino acids, distribution of which is independent from the phase or temperature of the starting materials. Only trace of amino acids can be found in these products before hydrolysis. The present results show that not only cosmic rays but also UV light are useful to form amino acid precursors in interstellar space.

\section{Energy Yields of Amino Acids from the SIM}

In the field of photochemistry, quantum yields were usually used. In order to compare energy yields of amino acids formed from the SIM by both UV and $\gamma$-irradiation, however, we will use the G-values, which is the number of formed molecules (after acid hydrolysis) per $100 \mathrm{eV}$ of given energy. Quantum yield in the present UV irradiation is approximately equal to the G-value multiplied by 0.05 . Fig. 2 shows the G-values of various amino acids by UV irradiation, when the SIM was irradiated as gaseous, liquid or solid (ice) mixtures. The G-values of glycine, the predominant amino acid, were around $10^{-2}$, which is independent from the phase of the SIM or the reaction temperature.

In the case of the $\gamma$-irradiation (dose rate: $\left.215 \mathrm{~Gy} \mathrm{~h}^{-1}\right)$, the $\mathrm{G}$-values of glycine $\left(\mathrm{G}_{\mathrm{Gly}}\right)$ was also ca. $10^{-2}$ in all the cases of the solid, liquid and gaseous phase irradiation. On the other hand, the G-value of glycine depended on the 


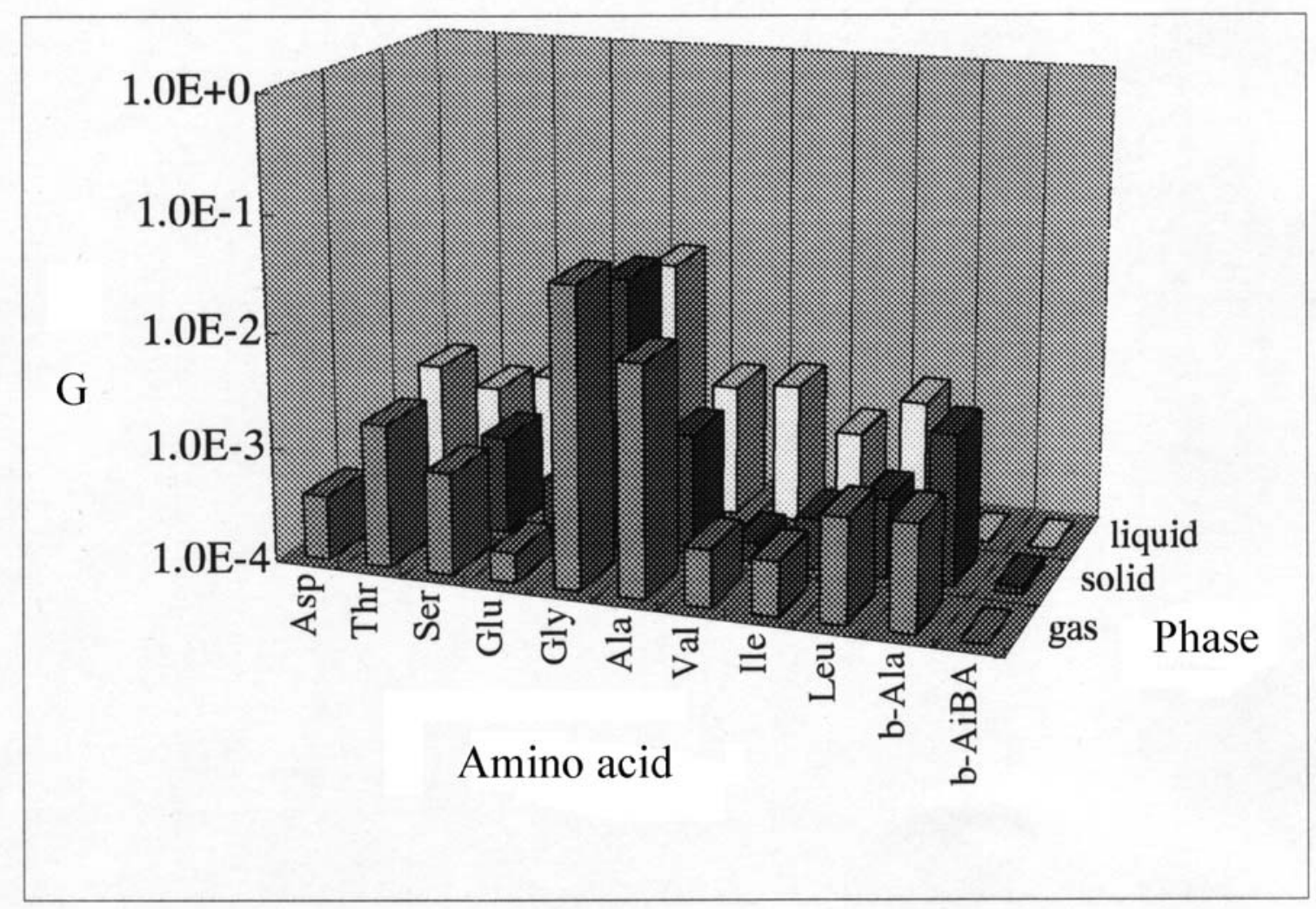

Fig. 2. G-Values of amino acids synthesized from the mixture of methanol, ammonia and water. Abbreviations are shown in the caption of Fig. 1.

dose rate: The higher the dose rate was, the lower the $\mathrm{G}_{\mathrm{Gly}}$ was.

Kasamatsu et al. (1997b) reported the $\mathrm{G}_{\mathrm{Gly}}$ from the same starting materials by irradiation with high-energy protons or electrons: The $\mathrm{G}_{\mathrm{Gly}}$ was larger in the case of gaseous phase irradiation than in the case of liquid or solid phase irradiation. The explanation for this is as follows: In the case of gaseous phase irradiation, the particle beams penetrated only a part of the gas mixture, so that most resulting product which rapidly escaped from the beam could avoid decomposition by further irradiation. In the case of solid/liquid phase irradiation, on the other hand, the resulting products would be decomposed by further irradiation since they could not move rapidly from the beams. In the present experiments by using photons, the whole of the mixture was subjected to irradiation with UV- or $\gamma$-photons. Thus the apparent G-value obtained was the difference of the G-value (formation) and the G-value (decomposition) in all of the three phases.

\section{Advantage of Exogenous Bioorganic Compounds}

The present results showed that amino acid precursors can be formed equally by cosmic radiation and UV, and the energy yield (G-value) is independent from the phases of starting materials. Here we discuss some advantages of exogenous bioorganic over endogenous bioorganic compounds.

\section{Formation of Amino Acid Precursors in ISDs vs. in primitive atmospheres}

Kasamatsu et al. (1997b) reported that amino acid precursors could be formed equally from such carbon compounds as carbon monoxide, methane and methanol by cosmic radiation. We can expect endogenous amino acid formation from carbon monoxide probably present in primitive earth atmosphere (Miyakawa et al., 2002). Since the yield of amino acid (precursors) depended on the molar ratio of carbon monoxide, only small amount of amino acids can be supposed to be formed if the molar ratio of carbon monoxide was quite low.

On the other hand, it is plausible that carbon monoxide, methane and methanol are sufficiently abundant in interstellar media. The present results suggest that amino acid precursors can be formed easily even the target is solid ice at quite low temperatures. 
Origin of Chiral Amino Acids

Since Pasteur discovered the asymmetry of life, many theoretical and experimental studies have been done to explain the origin of homochirality of biomolecules. Cronin and Pizzarello (1997) successfully detect enantiomeric excess of L-form amino acids in Murchison meteorite, which implies the origin of biomolecular chirality came from exogenous influence. There have been several scenarios of endogenous origin of biomolecular chirality: Radiation of specific circularly polarized light from neutron stars might result in asymmetric photosynthesis or photolysis (Bonner and Rubenstein, 1987). Possible asymmetric photolysis of amino acids by using circular polarized light from synchrotrons have been examined (Nishino et al., 2001; Takano et al., 2002).

\section{Formation of RNA Bases}

RNA bases are believed to be essential for the generation of life. Kobayashi and Tsuji (1997) showed that uracil, one of the four RNA bases, can be formed from simulated primitive atmosphere composed of carbon monoxide, nitrogen and water, by proton irradiation. Further investigations proved that several heterocyclic compounds such as orotic acid and nicotinic acid can be formed from the same starting materials (Miyakawa et al., 2002), but other nucleic acid bases having amino groups have not been identified among major products. Cytosine was identified in the hydrolysate of the product by proton irradiation of a mixture of carbon monoxide, ammonia and water (Yamanashi et al., 2001). It is suggested that ammonia is essential to obtain nucleic acid bases with amino groups. We have also identified thymine in the proton irradiation product from a mixture of methane, carbon monoxide and ammonia. On the other hand, adenine was reported to form from a mixture of methane, ammonia and water by electron irradiation (Ponnamperuma et al., 1963), but the other RNA bases with carbonyl groups have not been identified in it. Thus it can be said that reduced bases like adenine require reduced starting materials, and oxidized bases like uracil require oxidized starting materials. From the point of view above, the ISD environment seems to be more promising for the formation of RNA bases than primitive Earth atmosphere since ISDs have both reduced and oxidized components. Identification of purine bases in simulation experiments is required to confirm the present hypothesis.

\section{CONCLUSION}

The present results of laboratory simulation showed that amino acid precursors could be effectively formed in ice mantles of ISDs by cosmic radiation and ultraviolet light, though they are solid ices at low temperature. It was also suggested that the chirality of amino acids was generated by some cosmic relevance. Abiotic formation of several types of RNA bases required the presence of reduced and oxidized species in the starting materials: Ice mantles of ISDs are thus promising environments for it. We can summarize that bioorganic compounds endogenously formed in ISDs are significant sources for the first crucial building blocks of life on Earth.

It is also plausible that these exogenous organics were also supplied to the other planets than earth. If comets can bring sufficient kinds and amount of bioorganic compounds to planets, planetary biospheres might be generated, regardless of the composition of primary planetary atmospheres. It will be quite interesting to find and analyze cometary organics left in planets and satellites such as the Moon, Mars, Titan and Europa, as well as in cometary bodies themselves.

\section{ACKNOWLEDGMENTS}

The authors would like to thank Dr. H. Ikeda and Dr. D. Hiroishi, Research Center for Nuclear Science and Technology, University of Tokyo, for their kind help in ${ }^{60} \mathrm{Co}$ experiments. This research was supported in part by a Grant-In-Aid (No. 14340170) from MEXT (Ministry of Education, Culture, Sports, Science and Technology, Japan).

\section{REFERENCES}

Bernstein, M. P., J. P. Dworkin, S. A. Sandford et al., Racemic amino acids from the ultraviolet photolysis of 
interstellar ice analogues, Nature, 416, 401-403, 2002.

Bonner, W. A., and E. Rubenstein, Supernovae, neutron stars and biomolecular chirality, BioSystem, 20, 99-111, 1987.

Briggs, R., G. Ertem, J. P. Ferris et al., Comet Halley as an aggregate of interstellar dust and further evidence for the photochemical formation of organics in the interstellar medium, Origins Life Evol. Biosphere, 22, 287-307, 1992.

Chyba, C. F., and C. Sagan, Endogenous production, exogenous delivery and impact-shock synthesis of organic molecules: an inventory for the origins of life, Nature, 355, 125-132, 1992.

Cronin, J. R., and S. Pizzarello, Enantiomeric excesses in meteoritic amino acids, Science, 275, 951-955, 1997.

Greenberg, J. M., and A. Li, Silicate core-organic refractory mantle particles as interstellar dust and as aggregated in comets and stellar disks, Adv. Space Res., 19, 981-990, 1997.

Ikeda, H., and A. Suzuki, Radiolysis of n-dodecane and its physical property change based on the dose in one pass through a reference HA column, J. Nuclear Sci. Tech., 35, 697-704, 1998.

Kasamatsu, T., T. Kaneko, T. Saito, et al., Formation of organic compounds in simulated interstellar media with high energy particles, Bull. Chem. Soc. Jpn., 70, 1021-1026, 1997a.

Kasamatsu, T., T. Kaneko, T. Saito et al., Quantitative estimation of organic formation in interstellar dust environments, Chikyukagaku (Geochemistry), 31, 181-191, 1997b.

Kasting, J. F., Bolide impacts and the oxidation state of carbon in the earth's early atmosphere, Origins Life Evol. Biosphere, 20, 199-231, 1990.

Kissel, J., and F. R. Krueger, The organic component in dust from comet Halley as measured by the PUMA mass spectrometer on board Vega 1, Nature, 326, 755-760, 1987.

Kobayashi, K., T. Kaneko, T. Kobayashi et al., Analysis of products synthesized from simulated primitive planetary atmospheres. I. Amino acids, Anal. Sci., 7 suppl., 921-924, 1991.

Kobayashi, K., T. Kaneko, M. Tsuchiya, et al., Formation of amino acid precursors in cometary ice environments by cosmic radiation, Adv. Space Res., 16, 21-26, 1995.

Kobayashi, K., and T. Tsuji, Abiotic synthesis of uracil from carbon monoxide, nitrogen and water by proton irradiation, Chem. Lett., 1997, 903-904.

Kobayashi, K., T. Kaneko, T. Saito et al., Amino acid formation in gas mixtures by high energy particle irradiation, Origins Life Evol. Biosphere, 28, 155-165, 1998.

Kobayashi, K., T. Kaneko, A. Kouchi et al., Synthesis of amino acids in earth orbit: proposal, Adv. Space Res., 23, 401-404 (1999)

Kvenvolden, K., J. Lawless, K. Pering et al., Evidence for extraterrestrial amino-acids and hydrocarbons in the Murchison meteorite, Nature, 228, 923-926, 1970.

Miller, S. L., A production of amino acids under possible primitive earth conditions, Science, 117, 528-529, 1953.

Miyakawa, S., H. Yamanashi, K. Kobayashi, et al., Prebiotic synthesis from CO atmospheres: Implications for the origins of life, Proc. Nat. Acad. Sci. U.S.A., 99, 14628-14631, 2002.

Munoz Caro, G. M., U. J. Meierhenrich, W. A. Schutte et al., Amino acids from ultraviolet irradiation of interstellar ice analogues, Nature, 416, 403-406, 2002.

Nishino, H., A. Kosaka, G. A. Hembury et al., Mechanism of pH-dependent photolysis of aliphatic amino acids and enantiomeric enrichment of racemic leucine by circularly polarized light, Org. Lett., 3, 921-924, 2001.

Ponnamperuma, C., R. M. Lemmon, R. Mariner et al., Formation of adenine by electron irradiation of methane, ammonia and water, Proc. Nat. Acad. Sci. U.S.A., 49, 737-740, 1963.

Schlesinger, G., and S. L. Miller, Prebiotic synthesis in atmospheres containing $\mathrm{CH}_{4}, \mathrm{CO}$, and $\mathrm{CO}_{2}$. I. Amino acids, J. Mol. Evol., 19, 376-382, 1983,

Stocks, P. G., and A. W. Schwartz, Nitrogen-heterocyclic compounds in meteorites: significance and mechanisms of formation, Geochim. Cosmochim. Acta, 45, 563-569, 1981.

Takano, Y.m T. Kaneko, K. Kobayashi et al., Asymmetric potolysis of (DL)-isovaline by synchrotron radiation, in Program and Abstracts of 10th ISSOL Meeting and 13th International Conference on the Origin of Life, p. 92, Oaxaca, 2002.

Yamanashi, H., S. Takeda, K.-I. Murasawa et al., Identification and determination of nucleic acid bases abiotically formed in simulated planetary atmospheres, Anal. Sci., 17 Suppl., i1639-1642, 2001.

E-mail address of K. Kobayashiｋkensei@ynu.ac.jp

Manuscript received ; revised , accepted 